\title{
Motivational Profiles and Stages of Exercise Behavior Change
}

\author{
Hiroshi Matsumoto* and Koji Takenaka** \\ *Researcher, The Open Research-center Project of Mukogawa Women's University for Studying Lifestyle-related Diseases \\ (Doctoral Program, Graduate School of Human Sciences, Waseda University) \\ 6-46 Ikebirakicho, Nishinomiya, Hyogo 663-8558 Japan \\ hmatsu@mwu.mukogawa-u.ac.jp \\ ${ }^{* *}$ School of Human Sciences, Waseda University \\ 2-579-15 Mikajima, Tokorozawa, Saitama 359-1192 Japan \\ [Received August 15, 2003 ; Accepted February 3, 2004]
}

\begin{abstract}
The present study examined the relationship between exercise motivational profiles and stages of exercise behavior change. Four hundred and eighty-six Japanese adults participated in this study. In the first analysis, a cluster analysis identified four meaningful clusters in exercise motivational profiles. The first cluster was labeled the 'self-determined motivation profile' as the participants in this cluster had relatively high levels of intrinsic motivation and identified regulation. The second was the 'moderate motivation profile' with moderate scores on all measured motivational variables. The third was the 'nonself-determined motivation profile' as subjects in this group demonstrated higher nonself-determined motivation than self-determined motivation. The last cluster was the 'amotivation profile', which consisted of participants who had the highest scores in amotivation, and the lowest scores in intrinsic motivation and identified regulation. The second analysis explored how motivational profiles are related to stages of exercise behavior change. Participants in the first cluster were most likely to be classified in the maintenance stage. The results from this study provided the foundation for successful strategies to develop exercise adherence in adults focusing on motivational profiles.
\end{abstract}

Keywords: Motivation, Exercise, Physical activity, Self-determination theory, Stages of change

[International Journal of Sport and Health Science Vol.2, 89-96, 2004]

\section{Introduction}

The benefits of regular exercise to one's physical health are widely known. Besides its benefits to physical health, exercise is also effective for improvements to both mental health and quality of life [Takenaka, $(1998,1999)]$. However, although those benefits are recognized, it is not easy to continue regular exercise practices. In Japan, it is less than $30 \%$ of the total population those who engage in exercise longer than 30 minutes a day, more than twice a week for one year or longer period of time [Health, Labor and Welfare Ministry, Japan, (2002)]. Research has shown that approximately one half of the people who attend an exercise program drop out after several months [Carmody et al., (1980); Dishman, (1981, 1994, 2001); Franklin, (1988);
Oldridge, (1979); Ward and Morgan, (1984)]. On the basis of these studies, it is important to obtain the information to support one's continuous engagement in regular exercise.

The theories to alter one's health behaviors including exercise behaviors in the short term are relatively well known [Bellg, (2003)]. Those theories apply extrinsic reinforcements, such as penalty, compensation, behavioral and social reinforcement, and modeling to behavioral changes. However, it is difficult to maintain the long-term behavioral changes based solely on extrinsic reinforcements. Dunn and Rollnick (2003) state that the intrinsic motivation and the autonomous motivation are imperative to maintain preferable health behaviors in the long-term. Therefore, in order to maintain altered health behavior, one's motivation should be internalized, 
in that extrinsic motivation gradually shifts to an intrinsic emphasis.

In recent years, Deci and Ryan's $(1985,2000)$ "Self-Determination Theory (SDT)" has been a focus explaining internalization of motivation. In previous research, motivation has been considered as an innate endowment [Hayamizu, (1998)], and intrinsic and extrinsic motivations have been antithetically classified by dichotomy in that the intrinsic motivation is autonomous while extrinsic is heteronymous. From this perspective, motivation is seen to be static such character traits [Kurosawa, (1998)]. Additionally, the positive meaning is created only in the intrinsic motivation, while extrinsic is treated as undesirable. However, Self-Determination Theory hypothesizes that even extrinsically motivated behavior can occasionally change into intrinsically motivated behavior through the process of internalization. Additionally, extrinsic motivation is classified by the degrees of self-determination ${ }^{1}$. Therefore, in Self-Determination Theory, extrinsic motivation can be very similar to intrinsic motivation when motivation is changed from a nonself-determined to self-determined model ${ }^{2}$.

Conventional research has studied motivation as one single variable such as intrinsic motivation [Wang and Biddle, (2001)]. However, people often engage in exercise for enjoyment while they also engage in it for health reasons. Therefore, it is more appropriate to consider that their actual behaviors are reasoned by several motivations. Motivation should not be studies by the degree of a single type of motivation but should be studied by the pattern of the motivations. In order to understand actual motivation, the profile which strongly motivates behaviors need to be clarified [Vallerand and Fortier, (1998)]. Particularly in the field of physical activity research, Vallerand (1997) recommends that motivation be studied according to patterns. When motivation is studied by pattern, the self-determined motivation pattern (e.g., high in the intrinsic motivation and the identified regulation, and low in extrinsic regulation and amotivation) may be related to preferable cognition, emotion and behavior [Vallerand and Losier, (1999)].

Several studies examined the patterns of motivation using the Self-Determination Theory in the field of physical activity research. They examined youth participation in physical activity [Wang and Biddle, (2001)], physical education for youth [Ntoumanis, (2000)], and adult participation in sports [Vlachopoulos et al., (2000)]. However, research on exercise adherence based on the patterns of motivation has not yet been conducted. In previous studies on exercise adherence, even though various theories and models on the basis of behavioral sciences were applied, few studies incorporated the internalization of motivation By investigating the motivation patterns of exercise adherences using the Self-Determination Theory and exploring the relationship between motivational profiles ${ }^{3}$ and exercise adherence, it is possible to obtain valuable knowledge to support long-term exercise behavior changes.

This research examines the motivational profile of exercise behavior and exercise adherence among adults. The purpose of this research is, firstly, to illustrate the motivational profile for the regular exercise behavior. Secondly, this research aims to explore the relationship between extracted motivational profiles and the stages of exercise behavior change [Marcus and Simkin, (1993)], which indicate the process of the adoption and maintenance of a behavior.

1 With reference to Hayamizu (1998), this paper defines self-determination as " freely selecting satisfaction for one's own needs by oneself".

2 With reference to Matsumoto et al. (2003), the classification of motivation by SDT is explained as follows: amotivation is a condition lacking a sense of purpose and without an intention to act. This is seen when a person does exercises without any clear reasons to do so. Extrinsic motivation consists of subordinate concepts, or four behavior-regulating styles. They are extrinsic regulation, introjected regulation, identified regulation and integrated regulation. The degree of self-determination is seen to be higher according to this order. Extrinsic regulation is the stage where a person is not self-determined. An external force such as reluctantly doing exercise under doctor's orders starts all behavior. The introjected regulation is motivated by internal punishment or self-control such as feelings of guilt or embarrassment. The difference between the introjected and the extrinsic regulations is that a person in the stage of introjected regulation is seen to be self-determined to do exercises. In other words, this is the stage where the internalization of motivation has started. A person in the identify regulation identifies behaviors as his or her own values expressed as "I do exercise because this is important for me". Compared to introjected regulation, self-determination is positively promoted. Even if doing exercises is a measure of some kind for a person, he or she can do exercises in a more autonomous state if there is a consciousness of importance. The integrated regulation is the highest self-determined stage in the extrinsic motivation. In the integrated regulation, a person willingly does exercises seeing exercise as an avenue to reach a goal. Meanwhile, in intrinsic motivation, exercise itself is a purpose, and a person is motivated by fun or sense of satisfaction gained through exercise.

3 Referring to previous studies (Ntoumanis, 2002; Vlachopoulos et al., 2000; Wang and Biddle, 2001), the present research names the motivation pattern extracted by the cluster analysis as the "motivational profile". 


\section{Methods}

\subsection{Subjects}

Five hundred and forty people who consented to this research were investigated. They used exercise facilities around Tokyo, the participants to the exercise classes for health and the parents of students' in vocational schools. Participants who did not complete data were excluded, so 486 adults aged 18 or older (average $45.2 \pm 14.3$ years old, women $62.6 \%$, and effective respondent rate of $90 \%$ ) were analyzed. Their occupations are full-time workers $47.9 \%$, part-time worker $11.9 \%$, students $4.1 \%$, retired persons $8.6 \%$, housewives $17.7 \%$, others $8.8 \%$ and unknowns $0.8 \%$. Average education was 14.1 years $(\mathrm{SD}=2.1) .81 \%$ said the purpose of exercise was to maintain and improve their health.

\subsection{Contents}

\subsubsection{Exercise motivation}

This research used the self-determined motivation scale for exercise developed by Matsumoto et al. (2003). The self-determination motivation scale for exercise contains items based on Self-Determination Theory [Deci and Ryan, (1985, 2000)]. Validity measures (the factor validity by the explanatory factor analysis, the construct validity by the confirmatory factor analysis and the criterion-related validity by the discriminant analysis from the exercise adherence) and reliability measures (internal consistency and test-retest reliability) have been confirmed [Matsumoto et al., (2003)]. The scale includes five subscales consisting of intrinsic motivation (e.g. "Exercise itself is fun"), the identified regulation (e.g. "Exercise is a good way to improve myself"), the introjected regulation (e.g. "I will feel guilt if I do not exercise."), the external regulation (e.g. I exercise because other people such as a family member, a friend and a doctor say I should.") and the amotivation (e.g. "I don't know why I do exercises."). There were 18 items in total. The answer is chosen from five alternatives, namely "Quite true (5)", "Somewhat true (4)", "Neutral (3)", "Not necessarily true (2)" and "Not at all true (1)".

\subsubsection{Stages of the exercise behavior change}

Marcus and Simkin (1993) and Reed et al. (1997) have developed items to determine the stages of the exercise behavior changes. Referring to these items, a scale of five items was made [Oka et al., (2000) and Oka, (2003)], which the present research replicates. It consists of five items measuring actual exercise behavior performed both in the past and present time, and the condition of readiness to those exercise behaviors [Nigg and Riebe, (2002)]. The content of the items are as follows; "I currently do not exercise and do not intend to exercise in future (Precontemplation)." "I currently do not exercise, but I intend to exercise in the near future (in six months) (Contemplation)." "I currently get some exercise, but not regularly (Preparation)." "I currently exercise regularly, but I have only begun doing so within the past six months (Action)." "I currently exercise regularly and have been doing so for longer than six months (Maintenance)." The stages of the precontemplation and contemplation indicate the condition of readiness to the exercise behavior, and those of the preparation, action and maintenance suggest the condition of actual exercise behavior. "Regular exercise" in this case means exercise that is done for 20-30 minutes or longer at a time and repeated 2-3 times or more a week. From five alternatives, each subject selected one that is the most appropriate for his or her idea and behavior at the point. The validity of the scale was confirmed through research with college students [Oka et al., (2000)] and middle-aged adults [Oka, (2003)].

\subsection{Analysis method}

Firstly, in order to understand the motivational profile of the participants, the total scores of each factor in the scale of the self-determined motivation for exercise were converted to the standard score. According to these values, the cluster analysis was conducted using k-means method. Next, to study the relation between the motivational profiles found by the cluster analysis and the stages of exercise behavior change, $\chi^{2}$ test was run by making cross tabulations. Where a significant difference in $\chi^{2}$ test was found, the deviation of the number of people in the motivational profiles in each stage was examined using residual analysis. SPSS 11.0J for Windows was used for the statistical work mentioned above. 
Table 1 Descriptive statistics and internal consistency coefficents for overall sample

\begin{tabular}{lccc}
\hline & $\alpha$ & Overall means & $S D$ \\
\hline 1. Intrinsic motivation & .79 & 15.56 & 3.45 \\
2. Identified regulation & .78 & 16.23 & 3.22 \\
3. Introjected regulation & .84 & 8.72 & 3.95 \\
4. External regulation & .72 & 6.35 & 2.88 \\
5. Amotivation & .86 & 4.70 & 2.59 \\
\hline
\end{tabular}

\section{Results}

\subsection{Descriptive statistics and result of correlation analysis}

Table 1 indicates the internal consistency of each exercise motivation subscale of the participants and the descriptive statistics volume. In the subjects of this research, sufficient values were gained in the reliability of the scale ( $\alpha=.72-.86$ ), and correlation analysis was carried out for each variable. We can notice that the correlation coefficient between each subscale of motivation is strong when aspects of motivation are conceptually close. The closer motivational concept was the stronger correlation and vice versa (Table 2). Accordingly, the reliability of
Table 2 Correlation matrix for all variables

\begin{tabular}{|c|c|c|c|c|c|c|c|c|c|c|}
\hline & 1 & & 2 & & 3 & & 4 & & 5 & \\
\hline 1. Intrinsic motivation & & & & & & & & & & \\
\hline 2. Identified regulation & .62 & $* *$ & & & & & & & & \\
\hline 3. Introjected regulation & .25 & $* *$ & .32 & $* *$ & & & & & & \\
\hline 4. External regulation & -.05 & & .10 & $*$ & .34 & $* *$ & & & & \\
\hline 5. Amotivation & -.32 & $* *$ & -.42 & $* *$ & .21 & $* *$ & .31 & $* *$ & & \\
\hline 6. Stages of Change & .52 & $* *$ & .42 & $* *$ & .21 & $* *$ & .01 & & -.19 & ** \\
\hline
\end{tabular}

the scale was confirmed.

The distribution of the stages of exercise behavior change in this study is precontemplation (8\%), contemplation $(12.6 \%)$, preparation $(26.5 \%)$, action $(8 \%)$ and maintenance $(44.8 \%)$.

\subsection{Study of the exercise motivational profiles}

For the purpose of the study of the exercise motivational profiles in this research, the cluster analysis by k-means was conducted through setting 2-5 clusters. Consequently, it appears that the assortment by four clusters shows the profiles best. The characteristics of each cluster are shown in Figure 1. The first cluster (182 subjects, $37.4 \%$ overall) tends to be high in the self-determined

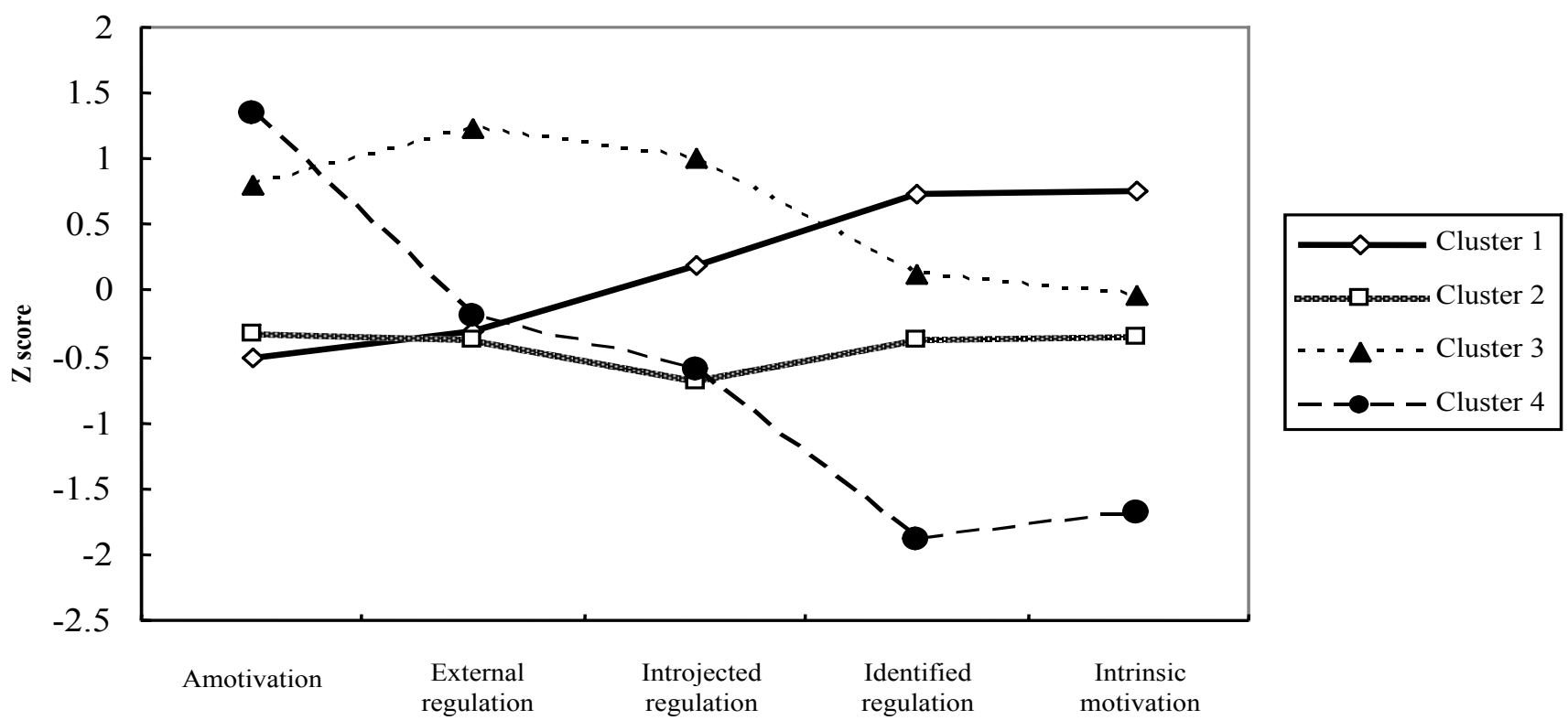

Figure 1 Cluster profiles for four cluster solution of K-means cluster analysis 
Table 3 Motivational profiles and Stages of Change

\begin{tabular}{|c|c|c|c|c|c|}
\hline \multirow[b]{2}{*}{ Motivational profiles } & \multicolumn{5}{|c|}{ Stages of change } \\
\hline & Precontemplation & Contemplation & Preparation & Action & Maintenance \\
\hline Cluster 1 & 2 & 12 & 32 & 16 & 120 \\
\hline "Self-determined motivation" & $-4.3^{* *}$ & $-3.1^{* *}$ & $-3.5^{* *}$ & 0.5 & $7.2^{* *}$ \\
\hline Cluster 2 & 11 & 25 & 62 & 9 & 48 \\
\hline "Moderate motivation" & -0.5 & 1.6 & $4.6^{* *}$ & -1.2 & $-4.2^{* *}$ \\
\hline $\begin{array}{l}\text { Cluster } 3 \\
\text { "Nonself-determined } \\
\text { motivation" }\end{array}$ & -1.7 & -0.3 & 0.5 & $2.0^{*}$ & -0.4 \\
\hline $\begin{array}{l}\text { Cluster } 4 \\
\text { "Amotivation" }\end{array}$ & $\begin{array}{c}22 \\
10.3^{* *}\end{array}$ & $2.8^{* *}$ & $\begin{array}{c}6 \\
-2.3^{*}\end{array}$ & -1.6 & $\begin{array}{c}6 \\
-4.7^{* *}\end{array}$ \\
\hline$\chi^{2}(120)=175.45, p<.01$ & & & & & ${ }^{*} p<.05, * * 1$ \\
\hline
\end{tabular}

motivation such as intrinsic motivation and the identified regulation, and low in the nonself -determined motivation. This cluster is called the "self-determined motivation profile" group. The second cluster (155 subjects, $31.9 \%$ overall) is called the "moderate motivation profile" group because all the motivations in the cluster have a tendency to show moderate values. In the third cluster (102 subjects, $21 \%$ overall), nonself-determined motivation is higher than the self-determined motivation. This group is called the "non-self-determined motivation profile". Lastly, the fourth cluster (47 subjects, 9.7\% overall) is high in the score of amotivation and low in other motivations. It is considered as the "amotivation profile" group.

\subsection{Relation between the exercise motivational profiles and the stages of exercise behavior change}

The relation between the four profiles displayed by the cluster analysis and the stages of exercise behavior change was explored. Table 3 displays the total of subjects in the stage that belongs to each motivational profile group. As a result of $\chi^{2}$ test, deviation of the number of the subjects is significant $\left(\chi^{2}(120)=175.45, p<.01\right)$. According to the residual analysis, in the self-determined motivation profile group, the subjects belonging to the precontemplation stage, contemplation stage and preparation stage is significantly few, while the subjects belonging to the maintenance stage is significantly many. In the moderate motivation profile group, the subjects belonging to the preparation stage were significantly many, and those belonging to the maintenance stage were significantly few. The nonself-determined motivational profile group shows the significant positive deviation in the action stage. Finally, the amotivation profile group is significantly high in the precontemplation and the contemplation, and significantly low in the preparation and the maintenance.

\section{Discussion}

This study aimed at clarifying the motivation profile of the exercise behavior by applying the Self-Determination Theory, and also exploring the relation between the extracted motivational profiles and the stages of exercise behavior change.

In order to examine the motivation patterns of exercise behavior as the first purpose, four clusters were extracted. The four clusters were; the group of "self-determined motivational profile" which was high in the intrinsic motivation and the identified regulation, that of "moderate motivational profile" in which all the motivations display moderate values, the "nonself-determined motivational profile" in which the nonself-determined motivation appeared to be higher than the self-determined motivation, and the "amotivation profile" which was high only in amotivation, and low in others. These cluster patterns had been identified in previous research [Ntoumanis, 
(2002); Vlachopoulos et al., (2000); Wang and Biddle, (2001)] that studied the motivation profile through Self-Determination Theory. Therefore, the four motivation patterns extracted in this research are considered as representative motivational profiles of exercise behavior.

Secondly, an aim was to explore the relation between the extracted motivational profiles and the stages of exercise behavior change. The results show that each motivational profile identifies its own characteristics toward the motivation behavior. In the self-determined motivational profile, subjects in the maintenance stage are significantly high in number, while subjects in the precontemplation, the contemplation and the preparation stages are significantly low. This may mean that those who are motivated intrinsically and feel that exercise is imperative for them have continued regular exercises for a long time at a level where they can appreciate the benefits to health. Therefore, it is suggested that where motivation for exercise behavior is internalized, there can be a strong possibility of exercise continuing for long period of time. The moderate motivational profile is significantly high in the preparation and low in the maintenance. We can consider people in the moderate motivational profile to not possessing nonself-determined or self-determined motivation. Though they do exercise, it is reasonable to suggest that they are not likely to develop their exercise behavior in the direction of regular exercises. The nonself-determination motivational profile group has strong extrinsic motivation and introjected regulation. This indicates the significant positive deviation in the number of people only in the action stage. In this profile, there seems to be many people who attempt to start exercises because of external pressure, for example a caution against inactivity given by a doctor. Yet the nonself-determination motivational profile does not show a significant deviation in the number of people in the maintenance. Taking this into consideration, the external pressure can change exercise behavior in the short-term, but it appears to be difficult to change long-term exercise behavior. Ingledew et al. (1998), who researched the relation between the exercise motivation and the stages of exercise behavior change, also demonstrated that extrinsic motivation was the highest in the action stage, and it tended to be low in the maintenance stage. The amotivation profile group displayed significant abundance in the precontemplation and contemplation stages, and significant small number in the preparation and the maintenance stages. As the amotivation profile group is considered to be unwilling to do exercise, this outcome may be logical.

The findings of this research clarify that internalization of motivation to exercise behavior plays an important part in maintaining behavior for long-term regular exercises. Primarily, the following would seem to be of importance; significant abundance is found in the preparation stage of the moderate motivational profile and the action stage of the nonself-determined motivational profile. If a person who has started doing regular exercises is in the category of the moderate motivational profile and the nonself-determined motivational profile, the key to maintenance of his or her long-term exercise behavior is whether motivation is internalized, and if there is intrinsic motivation. Deci and Ryan (2000) stated that satisfaction of psychological needs for competence, autonomy and relatedness facilitates natural growth processes including intrinsically motivated behavior and the integration of extrinsic motivations. Therefore, in order to maintain the intrinsic motivation and to promote the internalization of motivation, competence, autonomy and relatedness in exercise behavior should be strengthened. For example, it can be argued that positive feedback is effective in intensifying competence [Allen and Howe, (1998)], and autonomy is reinforced by through choice [Deci and Ryan, (1987); Matsumoto and Takenaka, (2003a)]. In addition, it is suggested that relatedness is intensified in group work and exercise programs in which participant's interaction is increased [Matsumoto and Takenaka, (2003b)]. However, more studies need to be carried out to ascertain the psychological factors that affect motivation to do exercise, since the previous findings are not sufficient.

Subsequently, an extraction method of subjects could be performed. In the present study, as many subjects were users of the exercise facilities, or the participants of exercise classes for health as well as being the parents of students' attending vocational schools, the generalizations from the findings are limited. Larger investigations using random sampling is be needed. Secondly, since this was a cross sectional study, the correlation between exercise motivation profiles and exercise behavior is not clear. The motivational profiles and exercise behavior 
targeted in this study are important at this point in the investigation, but they can change as time passes. Correlation needs to be clarified through longitudinal studies. Further studies could lead to new important outcomes through taking the above-mentioned challenges into consideration.

\section{Acknowledgment}

The authors are very grateful to Hideitsu Nakamura (Waseda University), Keiko Komuro (Waseda University), Masashi Yoshida (Waseda University), and Naoko Koezuka (University of Toronto).

\section{References}

Allen, J. B., and Howe, B. L. (1998) Player ability, coach feedback, and female adolescent athletes' perceived competence and satisfaction. Journal of Sport and Exercise Psychology 20: 280-299.

Bellg, A. J. (2003) Maintenance of health behavior change in preventive cardiology: Internalization and self-regulation of new behaviors. Behavior Modification 27: 103-131.

Carmody, T. P., Senner, J. W., Malinow, M. R., and Matarazzo, J. D. (1980) Physical exercise rehabilitation: long-term dropout rate in cardiac patients. Journal of Behavioral Medicine 3:163-168

Deci, E. L., and Ryan, R. M. (1985) Intrinsic motivation and self-determination in human behavior. Plenum: New York, NY.

Deci, E. L., and Ryan, R. M. (1987) The support of autonomy and the control of behavior. Journal of Personality and Social Psychology 53: 1024-1037.

Deci, E. L., and Ryan, R. M. (2000) The "What" and "Why" of goal pursuits: Human needs and the self-determination of behavior. Psychological Inquiry 11: 227-268.

Dishman, R. K. (1981) Biologic influences on exercise adherence. Research Quarterly for Exercise and Sport 52:143-159.

Dishman, R. K. (1994) Introduction: Consensus, problems and prospects. In: Dishman, R. K. (Ed.) Advances in exercise adherence. Human Kinetics: Champaign, IL, pp. 1-27.

Dishman, R. K. (2001) The problem of exercise adherence: Fighting sloth in nations with market economies. Quest 53: 279-294.

Dunn, C., and Rollnick, S. (2003) Lifestyle change. Elsevier limited: London.

Franklin, B. A. (1988) Program factors that influence exercise adherence: Practical adherence skills for clinical staff. In: Dishman, R. K. (Ed.) Exercise adherence: Its impact on public health. Human Kinetics: Champaign, IL, pp. 237-277.

Hayamizu, T. (1998) Jikokeisei no shinri: Jiritsutekidokizuke. Kaneko shobo: Tokyo. (in Japanese)

Ingledew, D. K., Markland, D., and Medley, A. R. (1998) Exercise motives and stages of change. Journal of Health Psychology 3: 477-489

Ministry of Health, Labour and Welfare, Japan. (2002) The national nutrition survey in Japan, 2000. Dai-ichi shuppan: Tokyo. (in Japanese)

Kurosawa, K. (1998) A dynamic theory of situation and internal process. In: Takuma, T. (Ed.) Handbook of Personality. Fukumura shuppan: Tokyo, pp. 97-103. (in Japanese)
Marcus, B. H., and Simkin, L. R. (1993) The stages of exercise behavior. Journal of Sports Medicine and Physical Fitness 33: 83-88.

Matsumoto, H., and Takenaka, K. (2003a) The relationship between the perceived exercise autonomy and the intention of exercise adherence. Japanese Journal of Health Promotion 5: 114-119. (in Japanese)

Matsumoto, H., and Takenaka, K. (2003b) The relationship between perceived exercise relatedness and exercise motivation, with mental health. Human Science Research 11: 147-160. (in Japanese)

Matsumoto, H., Takenaka, K., and Takaya, N. (2003) Development of the exercise motivation scale for exercise adherence based on Self-Determination Theory: The reliability and validity. Japanese Journal of Health Promotion 5: 120-129. (in Japanese)

Nigg, C. R., and Riebe, D. (2002) The transtheoretical model: Research review of exercise behavior and older adults. In: Burbank, P. M., and Riebe, D. (Eds.) Promoting exercise and behavior change in older adults: Interventions with the transtheoretical model. Springer Publishing Co: New York, NY, pp. 147-180.

Ntoumanis, N. (2002) Motivational clusters in a sample of British physical education classes. Psychology of Sport and Exercise 3: 177-194.

Oka, K., Takenaka, K., and Miyazaki, Y. (2000) Assessing the stages of change for exercise behavior among young adults: The relationship with self-reported physical activity and exercise behavior. Japanese Health Psychology 8: 17-23.

Oka, K. (2003) Reliability and validity of stages of change for exercise behavior scale among middle-aged adults. Japanese Journal of Health Promotion 5: 15-22. (in Japanese)

Oldridge, N. B. (1979) Compliance of post myocardial infarction patients to exercise programs. Medicine and Science in Sports and Exercise 11:373-375.

Reed, G. R., Velicer, W. F., Prochaska, J. O., Rossi, J. S., and Marcus, B. H. (1997) What makes a good staging algorithm: examples from regular exercise. American Journal of Health Promotion 12:57-66.

Takenaka, K. (1998) Exercise psychology: The contents and future in Japan. Japanese Journal of Sport psychology 25: 13-29. (in Japanese)

Takenaka, K. (2000) Exercise, sports and mental health. The Journal of Clinical Sports Medicine 17: 277-280. (in Japanese)

Vallerand, R. J. (1997) Toward a hierarchical model of intrinsic and extrinsic motivation. In: Zanna, M. (Ed.) Advances in experimental social psychology. Academic Press: New York, NY, pp. 271-360.

Vallerand, R. J., and Fortier, M. S. (1998) Measures of intrinsic and extrinsic motivation in sport and physical activity: A review and critique. In: Duda, J. L. (Ed.) Advances in sport and exercise psychology measurement. Fitness Information Technology: Morgantown, WV, pp. 81-101.

Vallerand, R. J., and Losier, G. F. (1999) An integrative analysis of intrinsic and extrinsic motivation in sport. Journal of Applied Sport Psychology 11: 142-169.

Vlachopoulos, S. P., Karageorghis, C. I., and Terry, P. C. (2000) Motivation profiles in sport: A self-determination theory perspective. Research Quarterly for Exercise and Sport 71: 387-397.

Wang, C. K., and Biddle, S. J. H. (2001) Young people's motivation profiles in physical activity: A cluster analysis. 
Journal of Sport and Exercise Psychology 23: 1-22.

Ward, A., and Morgan, W. P. (1984) Adherence patterns of healthy men and women enrolled in an adult exercise program. Journal of Cardiac Rehabilitation 4: 143-152.

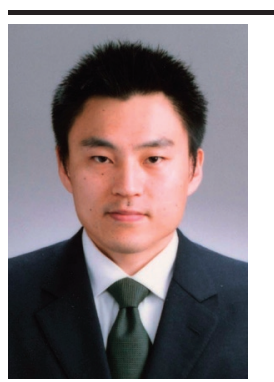

\section{Name:}

Hiroshi Matsumoto

\section{Affiliation:}

Researcher, The Open Research-center Project of Mukogawa Women's University for Studying Lifestyle-related Diseases

\section{Address:}

6-46 Ikebirakicho, Nishinomiya, Hyogo 663-8558 Japan

Brief Biographical History:

2001- Doctoral Program in Human Sciences, Waseda University 2004- Received Ph.D at Waseda University

2004- Researcher, The Open Research-center Project of Mukogawa Women's University for Studying Lifestyle-related Diseases

Main Works:

- "Development of the exercise motivation scale for exercise adherence based on Self-Determination Theory: The reliability and validity." Japanese Journal of Health Promotion, Vol.5, 120-129, (2003). (in Japanese)

Membership in learned Societies:

- The Japan Society of Physical Education, Health and Sport Sciences

- The Japanese Society of Sport Psychology

- The Japan Society of Health Promotion

- The Japanese Society of Behavioral Medicine 